MANNER OF DEATH

\title{
Variability of undetermined manner of death classification in the US
}

\author{
M J Breiding, B Wiersema
}

Injury Prevention 2006;12(Suppl II):ii49-ii54. doi: 10.1136/ip.2006.012591

See end of article for authors' affiliations

Correspondence to: Dr M J Breiding, Centers for Disease Control and Prevention, National Center for Injury Prevention and Control, 4770 Buford Highway, $\mathrm{NE}$, Mailstop K-60, Atlanta, GA, 30341 USA; dvi8@cdc.gov

Accepted 31 July 2006

\begin{abstract}
Objectives: To better understand variations in classification of deaths of undetermined intent among states in the National Violent Death Reporting System (NVDRS).

Design: Data from the NVDRS and the National Vital Statistics System were used to compare differences among states.

Main outcome measures: Percentages of deaths assigned undetermined intent, rates of deaths of undetermined intent, rates of fatal poisonings broken down by cause of death, composition of poison types within the undetermined-intent classification.

Results: Three states within NVDRS (Maryland, Massachusetts, and Rhode Island) evidenced increased numbers of deaths of undetermined intent. These same states exhibited high rates of undetermined death and, more specifically, high rates of undetermined poisoning deaths. Further, these three states evidenced correspondingly lower rates of unintentional poisonings. The types of undetermined poisonings present in these states, but not present in other states, are typically the result of a combination of recreational drugs, alcohol, or prescription drugs.

Conclusions: The differing classification among states of many poisoning deaths has implications for the analysis of undetermined deaths within the NVDRS and for the examination of possible/probable suicides contained within the undetermined- or accidental-intent classifications. The NVDRS does not collect information on unintentional poisonings, so in most states data are not collected on these possible/ probable suicides. The authors believe this is an opportunity missed to understand the full range of selfharm deaths in the greater detail provided by the NVDRS system. They advocate a broader interpretation of suicide to include the full continuum of deaths resulting from self-harm.
\end{abstract}

M uch of the difficulty in conceptualizing and measuring violent death lies in the implicit nature of intentionality, particularly for suicide. Perhaps stemming from this difficulty, a number of studies have concluded that suicides are underreported because of the misallocation of suicide verdicts to other manners of death, such as the "accidental" or undetermined classifications. ${ }^{1-6}$ Some have argued that the standard of proof (that is, typically "beyond a reasonable doubt") used by coroners/ medical examiners (C/ME) for legal reasons is a standard that is artificially high for public health purposes, and may result in the systematic exclusion of particular types of suicides. ${ }^{4} 7$ The difficulty of ascertaining a decedent's intent, combined with the legally defined burden of proof, likely restricts the full range of deaths due to self-harm from inclusion in violent death surveillance systems.

When intent cannot be established at the legally defined level, many possible or probable suicides are classified as undetermined in nature. ${ }^{1-4} 78$ General guidelines issued by the National Association of Medical Examiners state that the undetermined manner-of-death classification is to be used "when the information pointing to one manner of death is no more compelling than one or more other competing manners of death in thorough consideration of all available information". ${ }^{9}$ Of the 161269 resident injury deaths reported in the US in 2002, 3.0\% were of undetermined manner (intent). ${ }^{10}$ Despite deaths of undetermined intent comprising a relatively small percentage of injury deaths, disaggregating these data reveals troubling patterns and cause for concern.

of particular concern, the misclassification of suicides and homicides as deaths of undetermined intent results in underreporting of official suicide and homicide rates for certain demographic subgroups. ${ }^{8}{ }^{11-14}$ Further complicating matters, certain elements of a violent death may systematically influence whether a death is classified as undetermined in nature. These factors include: the mechanism of death, ${ }^{15-7} 15$ whether the decedent left explicit or circumstantial evidence of intention, ${ }^{1716}$ the quantity and quality of information available to the C/ME making the determination, ${ }^{14}{ }^{17}$ and differing criteria among C/MEs about the classification of certain types of deaths. ${ }^{18}$ While some of these factors are indicative of intentionality, each is independent of the decedent's true intentions and may result in a skewed picture of violent deaths.

Likely related to several of the aforementioned factors, considerable variability exists across both states and countries in terms of the frequency and rates of deaths of undetermined intent. ${ }^{10}{ }^{19}$ In many states, the undetermined category is used infrequently. In others, it represents a large proportion of violent deaths. As a cause of death, poisoning accounts for an increasingly large proportion of injury deaths of undetermined intent in the US. Between 1979 and 2002, the percentage of undetermined injury deaths due to poisoning rose from $32 \%$ to $69 \%$. More than half $(54 \%)$ of the undetermined-intent poisoning deaths in 2002 were due to narcotics and hallucinogens. ${ }^{10}$ Here, we suggest that difficulty in judging intentionality, coupled with differing C/ME philosophies on classifying manner of death, are largely responsible for variations among jurisdictions, particularly in cases of self-inflicted drug overdose (poisoning) deaths.

Abbreviations: NVDRS, National Violent Death Reporting System; WISQARS, Web-based Injury Statistics Query and Reporting System; $\mathrm{C} / \mathrm{ME}$, coroner/medical examiner. 
In addition to reviewing state-level variation in rates of manner-undetermined deaths, this research extends knowledge based on death certificates alone by taking advantage of added details on violent death investigations that are available from states participating in the US National Violent Death Reporting System (NVDRS). The NVDRS links data from C/MEs, police reports, death certificates, and like sources to produce a more detailed record of the characteristics and circumstances surrounding violent deaths, including those of undetermined manner or intent. We suspect, as have others, that variations across time and place in the amount and quality of information available describing the circumstances surrounding unnatural deaths likely have a direct impact on judgments of intent. ${ }^{14}$ We believe that such variations reduce the validity and reliability of statistics on violent death. At the same time, we argue that improved collection of accurate and detailed information on fatal injuries that result from high risk behaviors, regardless of the decedents' intentions, can lead to better and more effective violence prevention efforts.

\section{METHODS}

\section{Data sources}

We used two sources of data in this analysis to explore patterns of manner-undetermined death: the NVDRS and the Web-based Injury Statistics Query and Reporting System (WISQARS) which draws on mortality data from the US National Vital Statistics System. Standard descriptive statistics were used to compare groups. Two-sample $t$ tests, assuming equal variances, were computed to compare group means.

The NVDRS is a state-based, active surveillance system that collects information on all homicides, suicides, deaths of undetermined intent, deaths resulting from legal intervention (except legal executions), and deaths from unintentional firearm injuries. State health departments participating in the NVDRS typically identify these violent deaths as their death certificates are filed and then establish details of the cases from C/ME and law enforcement records. Details collected include the circumstances contributing to the deaths, interpersonal relationships, and toxicology results. Seven states (Alaska, Maryland, Massachusetts, New Jersey, Oregon, South Carolina, and Virginia) provided NVDRS data in 2003. Six additional states (Colorado, Georgia, North Carolina, Oklahoma, Rhode Island, and Wisconsin) provided NVDRS data in 2004. In 2003, these 13 states represented $23.4 \%$ of the US population and accounted for about $23.4 \%$ of the suicides, $22.7 \%$ of the homicides, and $42.1 \%$ of the manner-undetermined deaths. ${ }^{20}$

Violent deaths in the NVDRS are initially defined by International Classification of Diseases, 10th revision (ICD10) codes; NVDRS coders then review all pieces of evidence and assign a manner consistent with NVDRS coding practices. ${ }^{21}$ The manner of death used in the NVDRS analysis presented here is the abstractor-assigned manner of death. There are other differences in the NVDRS case definition, as noted elsewhere. ${ }^{21} 22$

The NVDRS makes a unique contribution to injury surveillance in that information about circumstances related to suicide, undetermined deaths, homicides, and unintentional firearm injuries are linked together from multiple sources in a single, incident-level database. The NVDRS does not collect information on unintentional deaths, aside from firearm related unintentional deaths, so detailed examination of these cases was not possible. Additional information about the NVDRS design, methods, and variable definitions are available elsewhere..$^{21} 22$

WISQARS data are collected by Centers for Disease Control (CDC)'s National Center for Health Statistics from death certificates registered in each of the 50 states and the District of Columbia. These data are compiled by CDC's National Center for Injury Prevention and Control from the National Vital Statistics System and made available online. ${ }^{20}$ Aggregated WISQARS data for 1999 through 2003 were calculated to compare NVDRS states with the remaining 50 states and the District of Columbia. Age adjusted rates were computed as deaths per 100000 residents using the 2000 standard population (for all races and both sexes). Rates represented an annual average computed over five years.

Violent deaths in WISQARS were identified by the following ICD-10 codes: deaths of undetermined intent (Y10-Y34, Y87.2, Y89.9), including undetermined-intent poisoning deaths (Y10-Y19); suicidal poisoning deaths (X60-X69); and unintentional poisoning deaths (X40-X49). These groupings are based on manner and cause information that are translated by nosologists from the manner of death checkbox and write-in cause of death blanks on death certificates filled in by C/MEs and other death certifiers. Although one might expect a great deal of agreement between the WISQARS/ICD-10 code intent groupings and NVDRS abstractor-assigned manner of death, it has not been established heretofore.

\section{RESULTS}

\section{Percentage of undetermined deaths by state}

Using NVDRS data, an examination of manner-undetermined deaths as a percentage of all violent deaths in each state reveals stark differences for several states (table 1). In $2003,60.0 \%$ (874 of 1456) of all violent deaths in Massachusetts were classified as manner-undetermined, while $42.4 \%$ (775 of 1829) of Maryland's deaths were classified similarly. The percentage of undetermined deaths in the remaining five NVDRS states was $7.0 \%$ (302 of 4292), with the largest percentage of undetermined death in Oregon (13.7\%, 116 of 844). In 2003, Maryland and Massachusetts comprised $84.5 \%$ (1649 of 1951) of manner-undetermined deaths within the NVDRS, but only 33.8\% (685 of 2023) of homicides and $26.4 \%$ (951 of 3603 ) of suicides.

The pattern in 2004 was similar even with the participation of six additional states in the NVDRS. In Massachusetts, $50.1 \%$ (635 of 1268) of all violent deaths were classified as manner-undetermined, while $38.1 \%$ (594 of 1560) of all deaths in Maryland were classified similarly. In addition, Rhode Island evidenced a high rate of undetermined death $(49.8 \% ; 120$ of 241$)$ in its first year of NVDRS participation. The rate of undetermined death in the remaining 10 NVDRS states for 2004 was $7.1 \%$ (718 of 10 135) with the largest rate in Oklahoma (17.3\%, 155 of 897). In 2004, Maryland, Massachusetts, and Rhode Island comprised 65.3\% (1349 of 2067) of the manner-undetermined deaths in the 13 states collecting data, but only $18.9 \%$ (710 of 3758) of homicides and $13.7 \%$ ( 1010 of 7379 ) of suicides.

\section{Rates of undetermined deaths in WISQARS}

To compare NVDRS data with the rest of the US and the District of Columbia, we analyzed WISQARS data from 1999 to 2003, the most recent years for which data were available. A summary of results found using WISQARS data is presented in table 2. From 1999 to 2003, Maryland, Massachusetts, and Rhode Island ranked first, third, and fourth (out of 51), respectively, in terms of the annualized average age adjusted rate of undetermined death. More important, the mean rate of undetermined-manner deaths in these three states (mean 9.21 per 100000 , SD 1.80; range 7.95-11.27) was statistically different from the mean rate (mean 1.25 per 100000 , SD 0.79 ; range $0.48-2.65$ ) in the other NVDRS states (difference in means 7.96 ; 95\% CI 6.44 to 9.48). 
Table 1 Number and percentage of deaths of undetermined manner within each state: National Violent Death Reporting System, 2003-04

\begin{tabular}{|c|c|c|c|c|}
\hline \multirow[b]{2}{*}{ State } & \multicolumn{2}{|l|}{2003} & \multicolumn{2}{|l|}{2004} \\
\hline & $\begin{array}{l}\text { Undetermined-manner } \\
\text { deaths, } \mathbf{n}(\%)\end{array}$ & $\begin{array}{l}\text { All violent } \\
\text { deaths* }\end{array}$ & $\begin{array}{l}\text { Undetermined-manner } \\
\text { deaths, } \mathbf{n}(\%)\end{array}$ & $\begin{array}{l}\text { All violent } \\
\text { deaths* }\end{array}$ \\
\hline Alaska & $5(2.8)$ & 176 & $5(2.5)$ & 201 \\
\hline Massachusetts & $874(60.0)$ & 1456 & $635(50.1)$ & 1268 \\
\hline Maryland & $775(42.4)$ & 1829 & $594(38.1)$ & 1560 \\
\hline New Jersey & $99(8.7)$ & 1135 & $4(0.4)$ & 895 \\
\hline Oregon & $116(13.7)$ & 844 & $102(12.8)$ & 798 \\
\hline South Carolina & $15(1.8)$ & 833 & $21(3.1)$ & 670 \\
\hline Virginia & $67(5.1)$ & 1304 & $73(5.6)$ & 1305 \\
\hline Colorado† & NA & NA & 109 (9.5) & 1144 \\
\hline Georgia† & NA & NA & 140 (8.4) & 1669 \\
\hline North Carolina† & NA & NA & $43(2.5)$ & 1696 \\
\hline Oklahoma† & NA & NA & 155 (17.3) & 897 \\
\hline Rhode Island† & NA & NA & $120(49.8)$ & 241 \\
\hline Wisconsint & NA & NA & $66(7.7)$ & 860 \\
\hline Totals & 1951 (25.7) & 7577 & 2067 (15.7) & 13204 \\
\hline \multicolumn{5}{|c|}{$\begin{array}{l}\text { *Includes homicide, suicide, legal intervention, unintentional firearm-related death, and deaths of undetermine } \\
\text { manner. } \\
\text { †Did not begin data collection until } 2004 \text {. } \\
\text { NA, not applicable. }\end{array}$} \\
\hline
\end{tabular}

Over the 1999-2003 period, $93.1 \%$ (5895 of 6331) of all manner-undetermined deaths in Maryland, Massachusetts, and Rhode Island were due to poisoning. Maryland, Massachusetts, and Rhode Island ranked first, third, and fourth, respectively, in their five-year annualized age adjusted rates for poisoning deaths of undetermined intent. More importantly, the mean rate of undetermined-intent poisonings in these three states (mean 8.55 per 100000 , SD 1.68; range 7.35-10.47) was significantly different from the mean rate (mean 0.64 per 100000 , SD 0.48; range 0.24-1.63) in the other NVDRS states (difference in means $7.92 ; 95 \%$ CI 6.70 to 9.13 ).

The large differences in rates between these two groups suggest that many undetermined-intent poisonings in Maryland, Massachusetts, and Rhode Island would have been classified as either suicides or as unintentional deaths had they occurred in other states.

We considered these possibilities in the two rightmost panels of table 2, comparing state rankings and rates of suicidal and unintentional poisonings with those discussed previously.

In contrast to their top ranks for undetermined-intent poisoning, Maryland, Massachusetts, and Rhode Island ranked 44th, 43rd, and 38th, respectively, in the rate of suicidal poisonings. However, the mean rate of suicidal poisonings in these three states (mean 1.51 per 100000 , SD 0.12 ; range 1.43-1.65) was not significantly different from the mean rate (mean 2.07 per 100000 , SD 0.64; range $1.32-3.37$ ) in other NVDRS states (difference in means $-0.562 ; 95 \%$ CI -1.40 to 0.27 ).

The pattern is even more pronounced for unintentional poisoning deaths during the same five-year period: Maryland, Massachusetts, and Rhode Island ranked 49th, 50th, and 51st, respectively. More important, the mean rate of unintentional poisonings in these three states (mean 0.99 per 100000 , SD 0.08; range $0.93-1.08$ ) was significantly different from the mean rate (mean 5.59 per 100000 , SD 1.74; range $4.01-10.27$ ) in the other NVDRS states

\begin{tabular}{|c|c|c|c|c|c|c|c|c|}
\hline \multirow[b]{3}{*}{ State } & \multirow{2}{*}{\multicolumn{2}{|c|}{$\begin{array}{l}\text { All undetermined- } \\
\text { intent deaths (Y10- } \\
\text { Y34, Y87.2, Y89.9) }\end{array}$}} & \multicolumn{6}{|c|}{ Poisoning deaths } \\
\hline & & & \multirow{2}{*}{\multicolumn{2}{|c|}{$\begin{array}{l}\begin{array}{l}\text { Undetermined intent } \\
\text { (Y10-Y19) }\end{array} \\
\begin{array}{l}\text { Age } \\
\text { adjusted } \\
\text { rate† } \quad \text { Rank } ¥\end{array}\end{array}$}} & \multirow{2}{*}{\multicolumn{2}{|c|}{ 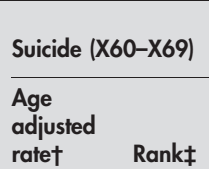 }} & \multicolumn{2}{|c|}{$\begin{array}{l}\text { Unintentional } \\
\text { (X40-X49) }\end{array}$} \\
\hline & $\begin{array}{l}\text { Age } \\
\text { adjusted } \\
\text { rate† }\end{array}$ & Rankł & & & & & $\begin{array}{l}\text { Age } \\
\text { adjusted } \\
\text { rate† }\end{array}$ & Rank \\
\hline Alaska & 2.65 & 7 & 0.91 & 20 & 2.13 & 20 & 10.27 & 3 \\
\hline Colorado & 2.33 & 12 & 1.63 & 10 & 3.37 & 4 & 6.20 & 16 \\
\hline Georgia & 1.00 & 32 & 0.34 & 42 & 1.32 & 46 & 4.78 & 29 \\
\hline Maryland & 11.27 & 1 & 10.47 & 1 & 1.43 & 44 & 1.08 & 49 \\
\hline Massachusetts & 8.40 & 3 & 7.84 & 3 & 1.45 & 43 & 0.97 & 50 \\
\hline New Jersey & 0.83 & 38 & 0.53 & 32 & 1.46 & 42 & 6.67 & 12 \\
\hline North Carolina & 0.48 & 49 & 0.24 & 48 & 1.98 & 23 & 5.76 & 19 \\
\hline Oklahoma & 1.07 & 27 & 0.48 & 33 & 2.47 & 12 & 6.33 & 14 \\
\hline Oregon & 2.06 & 13 & 1.27 & 13 & 2.50 & 10 & 4.96 & 26 \\
\hline Rhode Island & 7.95 & 4 & 7.35 & 4 & 1.65 & 38 & 0.93 & 51 \\
\hline South Carolina & 0.61 & 47 & 0.25 & 47 & 1.34 & 45 & 5.03 & 24 \\
\hline Virginia & 0.59 & 48 & 0.24 & 48 & 1.83 & 31 & 4.99 & 25 \\
\hline Wisconsin & 0.87 & 37 & 0.48 & 33 & 2.32 & 14 & 4.01 & 35 \\
\hline
\end{tabular}

*Accessed via WISQARS (see text).

†Average annual deaths per 100000 residents between 1999 and 2003; standard population is 2000, all races, both sexes.

‡Out of 51 (50 states and the District of Columbia). 
Table 3 Numbers and percentages of undetermined-intent deaths by type of poisoning: high rate undetermined-manner states $\checkmark$ low rate undetermined-manner states: NVDRS, 2003-04.

\begin{tabular}{|c|c|c|c|c|c|c|c|c|}
\hline \multirow[b]{2}{*}{ Type of poisoning } & \multicolumn{4}{|l|}{2003} & \multicolumn{4}{|l|}{2004} \\
\hline & MA/MD & $\begin{array}{l}\text { Other } \\
\text { states }\end{array}$ & Row total & $\begin{array}{l}\% \text { in } \\
M A / M D\end{array}$ & MA/MD/RI & $\begin{array}{l}\text { Other } \\
\text { states }\end{array}$ & Row total & $\begin{array}{l}\% \text { in } \\
M A / M D / R I\end{array}$ \\
\hline Recreational drugs only & 392 & 11 & 403 & 97.3 & 264 & 21 & 285 & 92.6 \\
\hline Alcohol only & 25 & 1 & 26 & 96.2 & 11 & 4 & 15 & 73.3 \\
\hline Prescription drugs only & 380 & 60 & 440 & 86.4 & 324 & 120 & 444 & 73.0 \\
\hline OTC drugs only & 8 & 5 & 13 & 61.5 & 7 & 2 & 9 & 77.8 \\
\hline Unknown drugs & 0 & 4 & 4 & 0.0 & 1 & 4 & 5 & 20.0 \\
\hline $\mathrm{CO}$ or other gas only & 4 & 3 & 7 & 57.1 & 2 & 9 & 11 & 18.2 \\
\hline Alcohol + recreational drugs & 65 & 2 & 67 & 97.0 & 49 & 1 & 50 & 98.0 \\
\hline Recreational drugs + prescription drugs & 117 & 4 & 121 & 96.7 & 117 & 10 & 127 & 92.1 \\
\hline Alcohol + prescription drugs & 71 & 7 & 78 & 91.0 & 67 & 5 & 72 & 93.1 \\
\hline Prescription drugs + OTC drugs & 16 & 5 & 21 & 76.2 & 12 & 4 & 16 & 75.0 \\
\hline Alcohol + recreational + prescription drugs & 14 & 1 & 15 & 93.3 & 20 & 0 & 20 & 100.0 \\
\hline Other specified substance & 7 & 1 & 8 & 87.5 & 25 & 3 & 28 & 89.3 \\
\hline Other combination of substances & 254 & 8 & 262 & 96.9 & 148 & 7 & 155 & 95.5 \\
\hline Not reported & 186 & 188 & 374 & 49.7 & 265 & 519 & 784 & 33.8 \\
\hline Unknown & 110 & 2 & 112 & 98.2 & 37 & 9 & 46 & 80.4 \\
\hline Total & 1649 & 302 & 1951 & 84.5 & 1349 & 718 & 2067 & 65.3 \\
\hline Total (in which poisoning was reported) & 1353 & 112 & 1465 & 92.4 & 1047 & 190 & 1237 & 84.6 \\
\hline
\end{tabular}

(difference in means $-4.91 ; 95 \%$ CI -7.19 to -2.62 ). This result, combined with the high rate of undetermined-intent poisonings in Maryland, Massachusetts, and Rhode Island, indicates that many undetermined poisonings in those states are likely being classified as unintentional poisonings in other NVDRS states. Figure 1 illustrates the inverse relationship between rates of undetermined-manner deaths and unintentional poisoning deaths among NVDRS states using WISQARS data.

\section{Poison types in the NVDRS}

According to 2003 NVDRS data, 1465 deaths of undetermined manner occurred in which a poison was noted in the record. Of these, 1353 (92.4\%) occurred in Maryland or Massachusetts. The same pattern was found in 2004; 1047 out of 1237 (84.6\%) undetermined poisonings were found in Maryland, Massachusetts, or Rhode Island. In 2003, six classifications of poisonings comprised most undetermined poisoning cases: 440 deaths due to prescription drugs only (86.4\% in Maryland/Massachusetts), 403 deaths due to

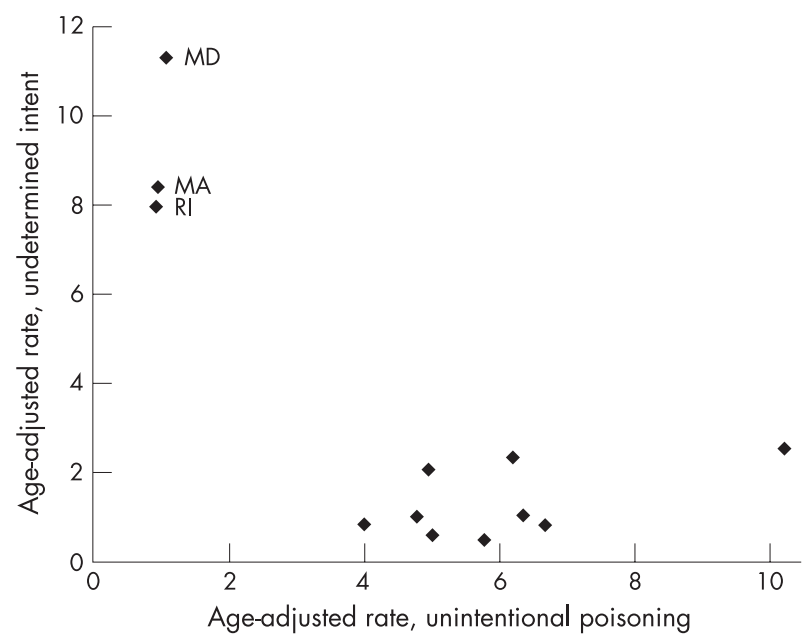

Figure 1 Scatterplot of age-adjusted rate of undetermined intent by the age adjusted rate of unintentional poisoning deaths among NVDRS States, 1999-2003 (source: WISQARS). MA, Massachusetts; MD, Maryland; RI, Rhode Island. street/recreational drugs only $(97.3 \%$ in Maryland/ Massachusetts), 262 deaths due to "other combination of substances" (96.9\% in Maryland/Massachusetts), 121 deaths due to street/recreational drugs and prescription drugs (97.0\% in Maryland/Massachusetts), 78 deaths due to alcohol and prescription drugs (91.0\% in Maryland/Massachusetts), and 67 deaths due to alcohol and street/recreational drugs $(97.0 \%$ in Maryland/Massachusetts). Results from this analysis of NVDRS data are summarized in table 3.

In 2004, these same six classifications of poisonings comprised most undetermined poisoning cases: 444 deaths due to prescription drugs only $(73.0 \%$ in Maryland/ Massachusetts/Rhode Island), 285 deaths due to street/ recreational drugs only (92.6\% in Maryland/Massachusetts/ Rhode Island), 155 deaths due to "other combination of substances" $\quad$ (95.5\% in Maryland/Massachusetts/Rhode Island), 127 deaths due to street/recreational drugs and prescription drugs (92.1\% in Maryland/Massachusetts/Rhode Island), 72 deaths due to alcohol and prescription drugs (93.1\% in Maryland/Massachusetts/Rhode Island), and 50 deaths due to alcohol and street/recreational drugs (98.0\% in Maryland/Massachusetts/Rhode Island).

The comparison of high rate versus low rate undeterminedmanner NVDRS states is complicated by differential reporting of poisoning type across the two groups. In 2003, Maryland and Massachusetts together reported type of poison in $82 \%$ (1353 of 1649) of cases while the five low rate undeterminedmanner states reported type of poison in only 37\% (112 of 302) of cases. In 2004, the addition of Rhode Island to the high rate group reduced poison type reporting to $78 \%$ (1047 of 1349) of cases while the reporting rate dropped to $26 \%$ ( 190 of 718 ) of cases in the low rate group of 10 states. Given this, computing significance tests of poison type differences between the two groups is not warranted until the question of differential reporting bias can be ruled out.

\section{DISCUSSION}

The number of deaths classified by NVDRS abstractors as undetermined in manner varies considerably among states participating in the NVDRS. This confirms patterns based on ICD-10 classifications found among the 50 US states and the District of Columbia. In particular, Massachusetts, Maryland, and Rhode Island tend to have more manner-undetermined deaths than other states. In addition, these states contribute 
a disproportionate percentage of the overall number of undetermined deaths within the NVDRS. Using WISQARS data, we found that these three states' poisonings comprise a large majority of the manner-undetermined deaths. Further, when comparing these states with all others and the District of Columbia, we found that they have a substantially higher rate of undetermined death and, more specifically, substantially higher rates of undetermined poisonings. We also found that the states with substantially higher rates of undetermined poisoning tend to have low rates of unintentional poisoning. Finally, NVDRS data suggest that the poisons involved in most undetermined poisoning deaths in Maryland, Massachusetts, and Rhode Island are some combination of recreational drugs, alcohol, or prescription drugs. The only undetermined poisoning deaths found in significant numbers in other NVDRS states were deaths in which prescription drugs alone were implicated, although differential reporting of poison types across states make this a very tentative finding.

The data indicate that C/MEs in Maryland, Massachusetts, and Rhode Island are more likely than C/MEs in other NVDRS states to classify a poisoning death as undetermined, particularly when a combination of prescription drugs, recreational drugs, or alcohol is implicated. We suspect, based on the WISQARS data, that in other NVDRS states similar poisonings were classified as unintentional. However, we were unable to examine this possibility directly using NVDRS because data on most unintentional deaths (that is, those that are not firearm related) are not collected by the system.

The discrepancies in the classification of poisoning deaths found here may reflect a lack of consensus among C/MEs. This is a critical issue for public health surveillance, particularly the NVDRS, because decisions about intent can significantly affect the validity of violent death data. Further, given the variation we found among states for poisoning deaths, variation in other types of deaths likely exists, albeit in less striking numbers. Future studies might examine this possibility. Future studies might also consider whether guidelines for manner-of-death classification disseminated by the National Association of Medical Examiners, particularly for the classification of poisoning deaths, can be made clearer.

This study's findings have implications for the analysis and interpretation of future studies examining undetermined deaths using NVDRS data. Because of the apparently discrepant criteria used for the classification of poisoning deaths in Maryland, Massachusetts, and Rhode Island, the data for undetermined deaths in these states are not directly comparable with other states' deaths of undetermined intent. Therefore, when analyzing undetermined deaths in the NVDRS, we recommend that researchers perform separate analyses: one for Maryland, Massachusetts, and Rhode Island, and another for the remaining states. An alternative strategy would be to analyze undetermined poisonings separately from other undetermined deaths. Performing separate analyses allows an examination of undetermined deaths which factors out the very large effect caused by differing criteria among states in classifying manner-undetermined poisonings.

Finally, even though the criteria Maryland, Massachusetts, and Rhode Island use in defining undetermined intent appear to be much more liberal than those of most other states, the increased number of undetermined deaths that they contribute to the NVDRS represents an excellent opportunity for studying a wider range of fatal self-injurious behaviors. Previous researchers have speculated that violent death surveillance systems significantly undercount suicide ${ }^{3}$ because C/MEs typically classify a death as suicide only when

\section{Key points}

- Significant variation exists among NVDRS states in the overall number and rates of manner-undetermined deaths; among those states with elevated numbers, most of the manner-undetermined deaths are due to poisoning.

- States that have many manner-undetermined poisoning deaths also tend to have few unintentional poisoning deaths, suggesting different criteria for the classification of poisoning deaths in some states.

- Results point to the need for consideration of a wider range of the continuum of intentionality, from those who leave evidence of suicidal intent to those who may be ambivalent and less likely to leave such evidence.

- Within the NVDRS, this might mean examining both manner-undetermined and unintentional deaths for possible/probable suicides.

- Results have implications for the analysis of mannerundetermined deaths in the NVDRS, namely that states with high rates of undetermined poisoning deaths should be analyzed separately from those with low rates.

significant evidence exists to indicate suicidal intent. However, many who die by suicide do not provide such evidence, particularly those who experience ambivalence about suicide. Little detail is known about people who die by suicide without a clear indication of intent because their deaths have typically been classified as undetermined or accidental in nature. However, those who experience ambivalence about suicide may provide the best opportunity for intervention given their doubts about taking their own life.

Previous researchers have discussed the need to examine the entire continuum of intentionality for self-injurious deaths. ${ }^{23}{ }^{24}$ Our study indicates that, within the NVDRS, such an examination would require looking not only at mannerundetermined deaths for possible/probable suicides, but also at some deaths classified as unintentional. In essence, it would require defining self-harm in a way that is less concerned with $\mathrm{C} / \mathrm{ME}$ verdict and more concerned with the behavior that resulted in the death. We therefore recommend future expansion of the NVDRS case inclusion criteria to cover all deaths involving self-harm, regardless of intent. While this may come at increased cost to the NVDRS system it is worth noting that Maryland, Massachusetts, and Rhode Island collect information on many, if not most, of these deaths already.

Until unintentional deaths can be examined for possible/ probable suicides, the unique collection of circumstance, toxicology, and situational variables of the NVDRS should be used to explore all available clues in deaths of undetermined intent. The many deaths of undetermined manner in Maryland, Massachusetts, and Rhode Island may provide an especially good opportunity to examine a wider portion of the continuum of intentionality among violent deaths.

\section{Authors' affiliations}

M J Breiding, Division of Violence Prevention, National Center for Injury Prevention and Control, Centers for Disease Control and Prevention, Atlanta, GA, USA

B Wiersema, Violence Research Group, Department of Criminology and Criminal Justice, University of Maryland, College Park, MD, USA 
This research was supported in part by a subcontract to the University of Maryland under Cooperative Agreement Number U17/CCU322392-

01 by the Centers for Disease Control and Prevention (CDC) to the Maryland Department of Health and Mental Hygiene (DHMH).

Competing interests: none.

Ethics approval: IRB approval was sought and obtained from the University of Maryland Human Subjects Committee prior to the collection of Maryland NVDRS data; however, no identifiable data were accessed or analyzed for this publication.

The findings and conclusions in this paper are those of the authors and do not necessarily represent the views of the Centers for Disease Control and Prevention, the Maryland Department of Health and Mental Hygiene, or the University of Maryland.

\section{REFERENCES}

1 Linsley KR, Schapira K, Kelly TP. Open verdict v. suicide: importance to research, Br J Psychiatry 2001;178:465-8.

2 Marušič A, Roškar S, Zorko M. Undetermined deaths: are they suicides? Croat Med J 2003:44:550-2.

3 Mohler B, Earls F. Trends in adolescent suicide: misclassification bias? Am J Public Health 2001;91:150-3.

4 O'Donnell I, Farmer R. The limitations of official suicide statistics. Br J Psychiatry 1995; 166:458-61

5 Ohberg A, Lonnqvist J. Suicides hidden among undetermined deaths. Acta Psychiatr Scand 1998;98:214-18.

6 Sorenson SB, Shen H, Kraus JF. Undetermined manner of death: a comparison with unintentional injury, suicide, and homicide death. Eval Rev 1997;21:43-57.

7 Lindqvist P, Gustafsson L. Suicide classification clues and their use: a study of 122 cases of suicide and undetermined manner of death. Forensic Sci Int 2002; 128:136-40.

8 Phillips DP, Ruth TE. Adequacy of official suicide statistics for scientific research and public policy. Suicide Life Threat Behav 1993;23:307-19.

9 Hanzlick R, Hunsaker JC, Davis GJ. A guide for manner of death classification, 1st edition. Atlanta, GA: National Association of Medical Examiners, 2002
10 Miniño AM, Anderson RN, Fingerhut LA, et al. Deaths: injuries, 2002. Natl Vit Stat Rep 2006;54:1-128.

11 Jason J, Carpenter MM, Tyler CW. Underrecording of infant homicide in the United States. Am J Public Health 1983;73:195-7.

12 Overpeck MD, Brenner RA, Trumble AC, et al. Infant injury deaths with unknown intent: what else do we know? Inj Prev 1999;5:272-5.

13 Sorenson SB, Shen H, Kraus JF. Coroner-reviewed infant and toddler deaths: many "undetermineds" resemble homicides. Eval Rev 1997;21:58-76.

14 Jobes DA, Berman AL, Josselson AR. Improving the validity and reliability of medical-legal certifications of suicide. Suicide Life Threat Behav 1987; 17:310-25.

15 Cantor C, McTaggart P, De Leo D. Misclassification of suicide: the contribution of opiates. Psychopathology 2001;34:140-6.

16 Lunetta P, Smith GS, Penttila A, et al. Undetermined drowning. Med Sci Law 2003:43:207-14.

$17 \mathrm{O}^{\prime}$ Carroll PW. A consideration of the validity and reliability of suicide mortality data. Suicide Life Threat Behav 1989;19:1-16.

18 Hanzlick R, Goodin J. Mind your manners. Part III: individual scenario results and discussion of the National Association of Medical Examiners manner of death questionnaire, 1995. Am J Forensic Med Pathol 1997; 18:228-45.

19 Fingerhut LA, Cox CS, Warner M. International comparative analysis of injury mortality: findings from the ICE on injury statistics. Adv Data 1998;303:1-19.

20 Centers for Disease Control and Prevention, National Center for Injury Prevention and Control. Web-based Injury Statistics Query and Reporting System (WISQARS). Available at http://www.cdc.gov/ncipc/wisqars (accessed February 2006).

21 National Violent Injury Statistics System; National Center for Injury Prevention and Control. National violent death reporting system coding manual [electronic resource]/produced under contract by the National Violent Injury Statistics System (NVISS), Harvard Injury Control Research Center, Harvard School of Public Health. Atlanta: National Center for Injury Prevention and Control, Centers for Disease Control and Prevention; 2003 Available at http://www.cdc.gov/ncipc/pub-res/nvdrs-coding/ Fullmanual.pdf (accessed February 2006).

22 Paulozzi L, Mercy J, Frazier L Jr, et al. CDC's National Violent Death Reporting System: background and methodology. Inj Prev 2004; 10:47-52.

23 Pompili M, Mancinelli I, Girardi $P$, et al. Toward a better understanding of equivocal deaths and suicides. Croat Med J 2004;45:103-4.

24 Shneidman ES. The psychological autopsy. Suicide Life Threat Behav $1981 ; 11: 325-40$. 